\title{
Avaliação das Funções Atentivas e Flexibilidade Mental em Dependentes Químicos
}

\section{Assessment of Attention Functions and Mental Flexibility in Drug Addicts}

\author{
Polyana Alvarenga Matumoto \& Joaquim Carlos Rossini* \\ Universidade Federal de Uberlândia, Uberlândia, Brasil
}

\begin{abstract}
Resumo
O presente estudo teve como objetivo investigar possíveis alterações na focalização atentiva e na flexibilidade mental de sujeitos diagnosticados com dependência química $(\mathrm{G} 1 ; n=20)$ quando comparado a um grupo controle que não faz uso nocivo de substâncias psicoativas (G2;n=20). Os participantes foram avaliados através do Teste Wisconsin de Classificação de Cartas (WCST) e um Teste de Desempenho Contínuo (TDC). Os resultados sugerem que os indivíduos do grupo G1 apresentaram um desempenho significativamente inferior no teste WCST e na maioria dos índices do teste TDC. Em conjunto, os desempenhos observados sugerem um déficit na flexibilidade cognitiva e um quadro de desatenção apresentado pelos participantes do grupo G1 em comparação ao grupo G2. Os achados são discutidos em termos de possíveis implicações para o tratamento de dependentes químicos.

Palavras-chave: Dependência química, atenção, Teste de Desempenho Contínuo, WCST, cognição.
\end{abstract}

\begin{abstract}
The present study aimed to investigate possible changes in attentive focus and mental flexibility of drug-addicted subjects $(\mathrm{G} 1, n=20)$ compared to a control group that does not make use of psychoactive substances $(\mathrm{G} 2, n=20)$. Participants were assessed using the Wisconsin Card Sorting Test (WCST) and a Continuous Performance Test (CPT). The results suggest that G1 individuals showed significantly lower performance on the WCST and in most CPT indexes. Altogether, the performances observed suggest a deficit in cognitive flexibility and a condition of inattention shown by $\mathrm{G} 1$ individuals in comparison to $\mathrm{G} 2$. The findings are discussed in terms of possible implications for the treatment of drug addicts.

Keywords: Drug addiction, attention, CPT, WCST, cognition.
\end{abstract}

O consumo de cocaína, álcool e Cannabis sativa (maconha) contribuem significativamente para o aumento de problemas graves relacionados à saúde pública, elevando os índices de violência, e acarretando uma série de complicações médicas e psiquiátricas.

De acordo com a Organização das Nações Unidas (Nations United Organization [ONU], 2012), o Brasil representou o segundo maior mercado de cocaína das Américas, apresentando um índice de consumo apenas abaixo do observado nos Estados Unidos. Para o mesmo período, os dados também apontaram um aumento significativo no consumo de maconha. Segundo um levantamento domiciliar sobre o uso de drogas realizado pelo Centro Brasileiro de Informações sobre Drogas Psicotrópicas da Universidade Federal de São Paulo (UNIFESP) em 2002, $11,2 \%$ dos brasileiros que vivem nas 107 maiores cidades do país são dependentes de álcool (Carlini, Galduróz, Noto, \& Nappo, 2002).

"Endereço para correspondência: Instituto de Psicologia, Universidade Federal de Uberlândia, Av. Pará, 1720, Campus Umuarama, Bloco 2C, Sala 2C-42, Uberlândia, MG, Brasil 38400-902. E-mail: jrossini@fapsi.ufu.br
A Classificação Internacional de Doenças (CID-10; World Health Organization, 1992) define como uso nocivo para a saúde o modo de consumo de uma substância psicoativa que é prejudicial à saúde. As complicações podem ser físicas ou psíquicas e o diagnóstico de Síndrome de Dependência pode ser estabelecido através de um conjunto de fenômenos comportamentais, cognitivos e fisiológicos que se desenvolvem após o uso repetido de uma substância psicoativa. Essa condição está tipicamente associada: (a) ao desejo intenso de consumir a droga, (b) à dificuldade de controlar o consumo, (c) à utilização persistente apesar das suas conseqüências prejudiciais, (d) a uma maior prioridade dada ao uso da droga em detrimento de outras atividades e obrigações, (e) a um aumento da tolerância pela droga e por vezes, (f) a um estado de abstinência física. O Manual Diagnóstico e Estatístico de Transtornos Mentais-IV (DSM-IV; American Psychiatric Association, 1994) define a dependência química como a presença de um agrupamento de sintomas cognitivos, comportamentais e fisiológicos indicando que o indivíduo continua consumindo uma substância, apesar de problemas significativos relacionados a seu uso. 
Vários estudos demonstram que processos cognitivos básicos estão envolvidos no comportamento de uso de drogas, no desenvolvimento da dependência e no processo de recaída (para uma revisão ver Rigoni, Susin, Trentini, \& Oliveira, 2013). Sendo assim, o estudo da cognição humana pode ser fundamental para a compreensão dos efeitos cognitivos e emocionais das substâncias psicoativas nos comportamentos aditivos. Estes estudos mostram que usuários nocivos e dependentes de substâncias psicoativas apresentam tendência a importantes alterações cognitivas, principalmente nas funções mnemônicas, executivas, atentivas e motoras (Andrade, Santos, \& Bueno, 2004; P. J. Cunha \& Novaes, 2004). De acordo com Verdejo-García, Lopez-Torrecillas, Arcos e Perez-Garcia (2005), os prejuízos neurológicos ocasionados pela dependência de uma determinada substância podem contribuir significativamente para a baixa adesão ao tratamento. Da mesma maneira, P. J. Cunha e Novaes (2004) sugerem que as alterações neurocognitivas têm implicação direta no tratamento, uma vez que déficits nas funções executivas podem gerar dificuldades em compreender e assimilar conceitos básicos da terapia, como traçar metas e cumprir objetivos que não envolvam uma recompensa imediata.

As funções executivas, de modo geral, são processos cognitivos superiores que permitem a manutenção de um funcionamento mental apropriado para alcançar um objetivo futuro, sendo responsável, em parte, pela capacidade de iniciar ações, planejar e prever meios de solucionar problemas, adiantar conseqüências e modificar estratégias de forma flexível (Grafman, 1999; Kristensen, 2006; Lezak, 1995; Lundqvist, 2005; Moss \& Kiliany, 1994). Essas funções permitem ao indivíduo desempenhar, de forma independente e autônoma, atividades dirigidas a um objetivo específico e englobam processos e comportamentos complexos. Estas ações dependem da integridade de vários processos cognitivos, emocionais, motivacionais e volitivos, os quais estão intimamente associados ao funcionamento dos lobos frontais (Almeida, Novaes, Bressan, \& Lacerda, 2008; Goldstein \& Volkow, 2002).

Segundo Bechara et al. (2001), o principal prejuízo nas funções executivas relacionado à dependência química é a correta representação de ganhos e perdas em ambientes aleatórios, nos quais o indivíduo precisa comparar as conseqüências diretas e indiretas de suas escolhas. Somado a este fato, vários estudos sugerem que indivíduos dependentes de substâncias apresentam déficits no desempenho em tarefas baseadas na inibição seletiva de uma classe de respostas preponderante (dificuldade de suprimir respostas habituais e automáticas em detrimento de um comportamento mais elaborado e adaptado) quando comparados a indivíduos não dependentes (Fillmore \& Rush, 2002; Finn, Mazas, Justus, \& Steinmetz, 2002; Forman et al., 2004; Kamarajan et al., 2005; Kaufman, Ross, Stein, \& Garavan, 2003; Li, Milivojevic, Kemp, Hong, \& Sinhá, 2006; Yucel \& Lubman, 2007).
De acordo com Verdejo-García, Bechara, Recknor e Pérez-García (2006), evidências crescentes indicam que indivíduos dependentes de substâncias psicoativas apresentam alterações no controle executivo. Nesse estudo, os autores compararam o desempenho de indivíduos dependentes de diferentes substâncias, como álcool, cocaína, metanfetamina e usuários de várias substâncias concomitantemente; com controles saudáveis através de uma bateria de testes comportamentais, cognitivos e emocionais. $\mathrm{O}$ grupo de indivíduos dependentes demonstrou um desempenho prejudicado nos testes cognitivos de memória de trabalho, resposta inibitória e flexibilidade mental, em comparação ao grupo controle. As medidas cognitivas, comportamentais e emocionais foram moderadamente correlacionadas.

Em um estudo recente, Amini, Alizadeh e Rezaee (2012) comparam o funcionamento das funções neurológicas - executivas de adultos dependentes químicos e adultos normais. Participaram desta pesquisa 38 adultos diagnosticados com dependência química, e 38 adultos que compunham o grupo controle que foram selecionados por amostra de conveniência. Os participantes foram examinados usando o Teste Wisconsin de Classificação de Cartas (WCST). Os resultados desse estudo sugerem uma diferença significativa nas funções executivas do grupo de dependentes químicos quando comparados ao grupo controle.

O objetivo da presente pesquisa foi investigar possíveis alterações em processos cognitivos de sujeitos que foram diagnosticados como dependentes de substâncias psicoativas como álcool, cocaína/crack e/ou maconha, e estavam em tratamento intensivo em caráter de internação-dia. Dentre as funções cognitivas avaliadas, destacam-se a focalização atentiva e a flexibilidade mental que foram mensuradas por um Teste Experimental Computadorizado de Desempenho Contínuo (TDC; Ballard, 2001; Riccio, Reynolds, Lowe, \& Moore, 2002; Salamat \& McPherson, 1999), e pelo Teste Wisconsin de Classificação de Cartas (WCST; J. A. Cunha et al., 2005).

\section{Método}

\section{Participantes}

A amostra pesquisada foi composta por dois grupos. O primeiro grupo (G1, $n=20$, sendo 17 do sexo masculino) foi formado por conveniência por sujeitos que foram diagnosticados como dependentes químicos (CID f10-f19) no processo de triagem do centro de tratamento intensivo em caráter de internação-dia em um setor de atenção à dependência química de uma universidade federal brasileira. As idades deste grupo variaram entre 22 e 58 anos $(M=43,5 ; D P=9,5)$. Quanto à escolaridade, $30 \%$ cursaram o ensino fundamental; $30 \%$ cursaram o ensino médio; $40 \%$ cursaram o ensino superior. Quanto às substâncias consumidas por esses participantes, $100 \%$ dos sujeitos eram usuários de álcool e $40 \%$ consumiam, 
concomitantemente, mais de uma substância (25\% álcool e cocaína; $25 \%$ álcool e maconha; $50 \%$ álcool, maconha e cocaína). O tempo de uso variou entre 1 e 32 anos $(M=$ $15,8 ; D P=11,55)$. Os critérios de exclusão para este grupo foram: (a) ter entre 18 e 60 anos; (b) abstinência da substância de dependência há, pelo menos, trinta dias; (c) ausência de indicações de alterações neurocognitivas decorrentes de outras especificações (avaliadas durante $o$ processo de triagem psiquiátrica); (d) escores normais na Escala Fatorial de Ajustamento Emocional / Neuroticismo (EFN; Hutz \& Nunes, 2001).

O segundo grupo (G2) constituiu o grupo controle, e foi composto por 20 sujeitos ( 12 do sexo masculino). As idades variaram entre 23 e 59 anos $(M=37,5 ; D P=13,7)$. Quanto ao grau de escolaridade, $20 \%$ cursaram o ensino médio e $80 \%$ cursaram o ensino superior. Os critérios de exclusão para este grupo foram: (a) ter entre 18 e 60 anos; (b) nunca ter consumido maconha e/ou cocaína/crack; (c) obedecer aos critérios de consumo de baixo risco para o álcool definidos pela Organização Mundial de Saúde (que definem como bebedores leves os homens que consomem menos que 21 unidades de álcool por semana e mulheres que consomem até 14 unidades de álcool por semana); (d) escores normais na EFN; (e) nunca ter apresentado queixas ou indicações de alterações neurocognitivas. Todos os participantes apresentavam visão normal ou corrigida. Todos os sujeitos $(n=40)$ foram informados dos objetivos da pesquisa e assinaram o termo de Consentimento Livre e Esclarecido antes da participação na avaliação.

\section{Instrumentos Utilizados}

A presente investigação utilizou a Escala Fatorial de Ajustamento Emocional / Neuroticismo (EFN; Hutz \& Nunes, 2001) para avaliar o estado emocional dos participantes em quatro dimensões: Escala de Vulnerabilidade, Escala de Desajustamento Psicossocial, Escala de Ansiedade e Escala de Depressão. O Teste de Classificação de Cartas Wisconsin (Heaton, Chelune, Talley, Kay, Curtiss, \& Cunha, 2005) foi utilizado para a avaliação do funcionamento dos lobos frontais e pré-frontais. Este teste tornou-se popular entre clínicos por ser sensível a disfunções cerebrais que afetam os lobos frontais, sendo classificado como uma medida do funcionamento "frontal" e "pré-frontal" (Heaton, Chelune, Talley, Kay, \& Curtiss, 1993). Este instrumento foi criado em 1948, posteriormente ampliado e revisado e tem o objetivo de avaliar o raciocínio abstrato e a capacidade do sujeito em gerar estratégias de solução de problemas, em resposta a condições de estimulação mutáveis (Berg, 1948; Heaton et al., 1993). O WCST é composto por 04 cartas-estímulo e 128 cartas-resposta. A tarefa do indivíduo é combinar as cartas-resposta com uma das quatro cartas-estímulo conforme uma das categorias (cor, forma, número, outra). A cada dez acertos sucessivos o critério é alterado, sem aviso prévio ao examinando, que apenas receberá um feedback se a classificação realizada está correta ou errada. O procedimento se repete até que o individuo complete seis séries corretamente classificadas ou termine o número de cartas. O levantamento dos dados é realizado estabelecendo escores quanto ao número total de erros; aos erros perseverativos, que diz respeito a quanto o sujeito persiste em responder a uma categoria específica que é incorreta; aos erros não perseverativos que consiste em uma resposta do sujeito de forma errônea por não combinar com o princípio perseverante ou vigente; $\mathrm{e}$ o percentual de repostas de nível conceitual, que denotam a compreensão do sujeito com os princípios corretos de classificação (J. A. Cunha, 2000; Heaton et al., 1993; Lezak, 1995). Dado ao reduzido número de estudos na população brasileira, na presente investigação, os escores brutos de cada categoria foram analisados.

Dentre os processos cognitivos, a atenção desempenha um importante papel nos quadros de dependência e no abuso crônico de substâncias (Peuker, Lopes, Menezes, Cunha, \& Bizarro, 2013). Esse aspecto da cognição foi investigado através do Teste de Desempenho Contínuo (TDC). Este tipo de instrumento avalia a capacidade de inibir uma resposta preponderante e o tempo de reação a um estímulo imperativo por meio de tarefas de caráter GO/NOGO. Este tipo de tarefa demanda uma resposta do participante na presença de um estímulo alvo (GO) e uma não-resposta (inibição comportamental) na presença de um estímulo não-alvo (NOGO). Desempenhos abaixo do esperado neste tipo de tarefa podem evidenciar comprometimentos no sistema atentivo e mnemônico (desatenção, impulsividade e déficit na memória operacional; Dougherty et al., 2003; Moeller et al., 2005). A apresentação dos estímulos e o registro do tempo de reação dos participantes foram realizados diretamente no computador pelo utilitário E-prime 2.0 (Schneider \& Zuccoloto, 2007). Os sujeitos realizaram uma tarefa de caráter "GO-NOGO" baseada em uma pesquisa anterior realizada por Hester e Garavan (2004). Cada prova era iniciada com a apresentação de um ponto de fixação que permanecia na tela por $1000 \mathrm{~ms}$. Imediatamente após a apresentação da fixação uma letra maiúscula ou um número era apresentado no centro da tela por $100 \mathrm{~ms}$. Imediatamente após a apresentação do estímulo alvo, uma máscara composta pelo símbolo "\#”, na cor branca, e disposto em quatro colunas e três linhas, era apresentada até a resposta do participante, ou pelo tempo limite de $1000 \mathrm{~ms}$. Após a resposta do sujeito, os símbolos que compunham a máscara eram apresentados na cor azul (resposta correta) ou vermelha (resposta incorreta), por um intervalo de $1500 \mathrm{~ms}$. Imediatamente após este intervalo uma nova prova era iniciada, como mostra a Figura 1.

A tarefa dos participantes era pressionar a tecla " $\mathrm{n}$ " do teclado numérico, o mais rápido possível, com a apresentação de uma letra. Quando um número era apresentado, o participante foi instruído a não efetuar nenhuma resposta. Cada participante realizou 100 provas, destas 55\% eram letras e $45 \%$ eram números. A sessão experimental apresentava uma duração média de 10 minutos. 


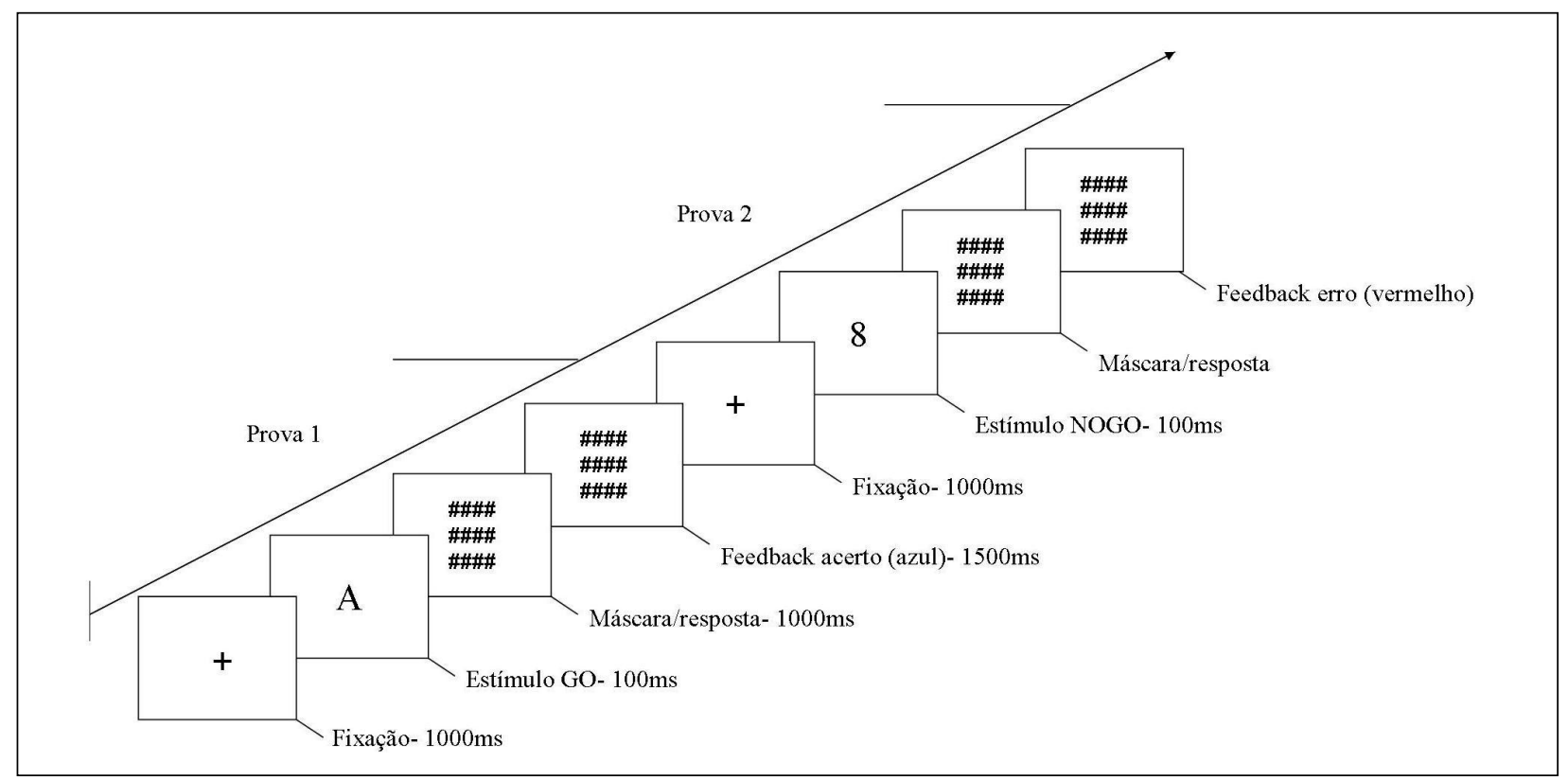

Figura 1. Representação esquemática da sucessão de provas no teste TDC.

\section{Procedimentos}

A primeira sessão foi destinada a realização de uma entrevista sócio-demográfica onde foram coletados dados como: idade, sexo, consumo, droga de escolha, padrão de uso, tempo de abstinência, nível sócio-econômico, grau de escolaridade, anamnese (história anterior de síndromes psiquiátricas e alterações neurocognitivas não relacionadas ao abuso de substâncias); e a aplicação da EFN. Caso o participante preenchesse os requisitos exigidos, os testes WCST e o TDC foram realizados, em duas sessões de aproximadamente 30 minutos para cada teste. Os escores do WCST foram analisados utilizando-se o teste estatístico Mann-Whitney. O desempenho dos participantes no TDC foi analisado através do teste $t$ de Student para amostras independentes. O nível de significância estatístico adotado para todas as análises foi igual a 0,05 .

\section{Resultados}

O teste $U$ de Mann-Whitney para amostras independentes foi utilizado para a análise da distribuição das idades dos grupos e não evidenciou diferença significativa $U=$ $155(z=1,22) ; p=0,22$. O desempenho dos participantes no WCST em função dos fatores demográficos gênero e escolaridade foram analisados através do teste Kruskal-Wallis para grupos independentes que não evidenciou diferenças significativas tanto no grupo G1 [gênero $H$ $(1,20)=1,80 ; p=0,67$; escolaridade $H(2,20)=3,81 ; p$ $=0,15$ ] quanto no grupo G2 [gênero $H(1,20)=0,93 ; p=$ $0,33$; escolaridade $H(1,20)=1,08 ; p=0,30]$.

Como medida do funcionamento executivo, os escores brutos dos sujeitos no WCST foram analisados, comparando os grupos G1 e G2 através do teste $U$ - Mann Whitney, nas categorias sumariadas na Tabela 1 .
Nesta tabela, verifica-se que houve diferença estatisticamente significativa entre os grupos G1 e G2 em todas as categorias analisadas. Estes resultados sugerem que os participantes diagnosticados em dependência química (G1) apresentam menor flexibilidade na resolução de problemas e funcionamento executivo rebaixado quando comparados a indivíduos do grupo controle (G2).

Para avaliar as funções atentivas dos sujeitos, os resultados do TDC foram comparados através do teste $t$ de Student para amostras independentes, como mostra a Tabela 1. Esta análise mostrou que o tempo de reação do grupo G1 foi significativamente superior ao do grupo G2. O tempo de reação do erro cometido pelos participantes também apresentou uma diferença significativa entre os grupos investigados. Em conjunto, esses resultados sugerem uma lentificação no grupo G1 em relação ao grupo G2. Os erros cometidos foram classificados em omissões e comissões. O resultado desta análise corrobora a interpretação que o grupo G1 apresentou um estado de desatenção significativamente superior ao observado no grupo G2. Os resultados são sumariados na Tabela 1.

\section{Discussão}

A presente investigação evidenciou características cognitivas importantes de uma amostra de sujeitos diagnosticados em dependência química (G1) e avaliados através do teste Wisconsin de Classificação de Cartas (WCST) e um teste computadorizado de Desempenho Contínuo (TDC). Apesar de não ter sido possível estabelecer grupos específicos de participantes em função de uma única substância de abuso, uma vez que todos os participantes do grupo G1 eram usuários de álcool e $40 \%$ destes consumiam mais de uma substância concomitantemente, os 
Matumoto, P. A. \& Rossini, J. C. (2013). Avaliação das Funções Atentivas e Flexibilidade Mental em Dependentes Químicos.

Tabela 1

Escores Médios de Desempenho do Grupo G1 e G2 no Teste WCST e TDC

\begin{tabular}{|c|c|c|c|c|c|c|c|}
\hline & \multirow{2}{*}{$\begin{array}{c}\mathrm{G} 1(n=20) \\
M \pm D P\end{array}$} & \multirow{2}{*}{$\begin{array}{c}\mathrm{G} 2(n=20) \\
M \pm D P\end{array}$} & \multicolumn{5}{|c|}{ Significância } \\
\hline & & & $U$ & $/ \quad z$ & $/ r$ & $r$ & $p$ \\
\hline \multicolumn{8}{|l|}{ WCST } \\
\hline Total de erros & $49,9 \pm 28$ & $17,5 \pm 11$ & 72,5 & $-3,45$ & &, 55 & $<0,01$ \\
\hline Erros perseverativos & $30,1 \pm 18$ & $10,6 \pm 7$ & 73,0 & $-3,44$ & &, 54 & $<0,01$ \\
\hline Erros não perseverativos & $20,1 \pm 18$ & $6,7 \pm 48$ & 4,5 & $-3,13$ & &, 49 & $<0,01$ \\
\hline Número de ensaios & $115,2 \pm 21$ & $89,7 \pm 18$ & 91,0 & $-3,04$ & &, 48 & $<0,01$ \\
\hline Respostas de nível Conceitual & $44,2 \pm 27$ & $76,5 \pm 9$ & 76,5 & $-3,34$ & &, 53 & $<0,01$ \\
\hline TDC (TR) & $M(\mathrm{~ms}) \pm D P$ & $M(\mathrm{~ms}) \pm D P$ & $t$ & l & $d /$ & & $p$ \\
\hline Tempo de reação (acertos) & $417 \pm 100$ & $332 \pm 64$ & 3,14 & & ,03 & & $<0,01$ \\
\hline Tempo de reação (erros) & $358 \pm 142$ & $282 \pm 86$ & 2,06 & & 67 & & 0,04 \\
\hline \multicolumn{8}{|l|}{ TDC (erros) } \\
\hline Comissões & $7,55 \pm 12$ & $3,25 \pm 3,5$ & 1,51 & & ns & & ns \\
\hline Omissões & $3,25 \pm 2,4$ & $1,35 \pm 1,7$ & 2,80 & &, 90 & & $<0,01$ \\
\hline Total de Erros & $10,8 \pm 12$ & $4,6 \pm 4,4$ & 2,05 & &, 72 & & $<0,05$ \\
\hline
\end{tabular}

Nota. Adequação do $n$ da amostra para o TDC (TR): $n=\left(\sigma^{2} \mathrm{G}_{1}+\sigma^{2} \mathrm{G}_{2}\right)(\mathrm{z} \alpha+\mathrm{z} \beta)^{2} / d^{2}$ (Campana, 1995). $\sigma^{2} \mathrm{G}_{1}=100^{2} ; \sigma^{2} \mathrm{G}_{2}=64^{2} ; \mathrm{z} \alpha$ $=1,65 ; \mathrm{z} \beta=1,28 ; d=M\left(\mathrm{TRG}_{1}\right)-M\left(\mathrm{TRG}_{2}\right)=85^{2} . n$ mínimo estimado por grupo $=17$.

resultados observados sugerem um prejuízo significativo na flexibilidade mental e nos processos atentivos deste grupo, quando comparados a um grupo controle.

A dependência química pode ser definida como um estado psicológico persistente no qual o sujeito apresenta uma capacidade reduzida de regular um comportamento compulsivo em relação a uma substância, apesar das conseqüências não adaptativas frente à demanda ambiental (Feil et al., 2010). Os resultados observados no WCST sugerem uma maior rigidez de pensamento e critérios no grupo G1. Isto é evidenciado por um número significativamente superior de erros perseverativos neste grupo, ou seja, a adoção e manutenção de um critério equivocado de classificação por um número maior de tentativas, a despeito do indicativo ambiental de inadequação do critério (feedback do aplicador). Este resultado pode caracterizar uma dificuldade por parte do grupo G1 em identificar as estratégias mais adequadas em resposta a uma situação de escolha ou da resolução de problemas que exigem flexibilidade do raciocínio (Bolla, Brown, Eldreth, Tate, \& Cadet, 2002; P. J. Cunha \& Novaes, 2004). Somado a estes fatos, a ocorrência de um grande número de erros perseverativos pode ser considerado um importante marcador comportamental para disfunções relacionadas ao córtex pré-frontal (Heaton et al., 1993; Strauss, Sherman, \& Spreen, 2006; Woicik et al., 2011). O grupo G1 também apresentou um número significativamente maior de erros não perseverativos, o que sugere a adoção, não criteriosa, de uma lógica de classificação incorreta. Tal desempenho sugere um estado de desatenção caracterizado por uma dificuldade em manter o foco da atenção na execução de uma tarefa proposta.
Outro aspecto importante analisado foi o número de respostas de nível conceitual. Tais respostas indicam possíveis insights acerca dos princípios corretos de classificação._Novamente, o grupo G1 apresentou um desempenho inferior quando comparado ao grupo controle G2 Tal resultado pode ser o reflexo de déficits no sistema mnemônico (Aharonovich et al., 2006; Amini et al., 2012; Tinius, 2003; Turner, LaRowe, Horner, Herron, \& Malcolm, 2009; Woicik et al., 2009), uma vez que prejuízos no funcionamento da memória operacional e na correta recuperação e integração da informação podem contribuir para um padrão de respostas desadaptadas. Este padrão de respostas inadequadas pode ser aplicado a diversas situações do cotidiano, bem como nos comportamentos aditivos, o que traz implicações importantes para o desenvolvimento de estratégias de intervenção terapêuticas eficazes. No entanto, uma limitação importante do presente estudo foi o número reduzido de participantes ( 20 participantes em cada grupo), uma vez que a amostra foi composta por conveniência.

O desempenho dos dois grupos investigados no TDC corrobora os resultados observados no WCST. As características atentivas dos participantes foram avaliadas sob os seguintes aspectos: (a) tempo de reação para a resposta; (b) tempo de reação do erro; (c) número total de erros cometidos; (d) número de omissões (respostas incorretamente não efetuadas); (e) número de comissões (respostas efetuadas incorretamente). A análise do tempo de reação, tanto para as respostas corretas quanto para as respostas incorretas, evidenciou uma lentificação dos participantes do grupo G1 em relação ao grupo controle. Este aumento no tempo de reação observado no grupo de dependentes químicos 
é um importante indicativo de desatenção (Riccio et al., 2002). Esta interpretação é corroborada pelo número significativamente superior de omissões observado no grupo G1 em relação à quantidade de erros deste tipo cometido pelo grupo G2. O número de comissões cometidas não foi significativamente distinto entre o grupo G1 e G2. Este fato sugere que o controle inibitório apresentado pelo grupo G1, frente a um estímulo imperativo, não difere do nível de controle inibitório apresentado pelo grupo G2. Muitos estudos têm apontado para um comprometimento deste aspecto do processamento atentivo em dependentes químicos, sobre tudo em usuários crônicos de cocaína (Connolly, Foxe, Nierenberg, Shpaner, \& Garavan, 2012; Fillmore \& Rush, 2002; Goldstein, Volkow, Wang, Fowler, \& Rajaram, 2001; Kaufman et al., 2003). A não observância deste aspecto no desempenho do grupo G1 pode ser explicada por um número de fatores. $\mathrm{O}$ grupo investigado foi composto por poliusuários em diferentes períodos de abstinência (entre trinta dias e vários anos), o que pode sugerir comprometimentos distintos da função inibitória em diferentes períodos de abstinência (Connolly et al., 2012). Neste sentido, o presente estudo limitou-se a uma avaliação, através da EFN, de apenas alguns aspectos da personalidade, não investigando possíveis transtornos relacionados ao Eixo I do DSM-IV. Estes aspectos serão investigados em delineamentos futuros. Outro aspecto que pode ter influenciado o desempenho dos participantes talvez tenha sido a neutralidade emocional dos estímulos apresentados (letras e números). Uma hipótese interessante, demonstrada nos achados de Noel et al. (2005) é que estímulos com significado emocional associado à substância de consumo sejam mais efetivos em eliciar comportamentos impulsivos.

Estes aspectos são importantes para o planejamento da intervenção terapêutica nesta população, uma vez que prejuízos neurológicos podem contribuir para a baixa adesão ao tratamento (Verdejo-García et al., 2005). Porém, as implicações para o tratamento destes pacientes são mais abrangentes. As próprias técnicas de intervenção podem ser ineficazes frente à dificuldade destes sujeitos em reter novas aprendizagens e manter um bom nível atentivo durante as atividades propostas (Cunha, Peuker, \& Bizarro, 2012). Somado a este fato, a dificuldade em resolver problemas, considerando diferentes alternativas, e a redução da flexibilidade cognitiva que dificulta a adoção de novos comportamentos mais adaptados, apresenta uma importância fundamental, tanto para o planejamento das intervenções quanto para o prognostico de sucesso quanto à adesão ao tratamento.

\section{Referências}

Aharonovich, E., Hasin, D. S., Brooks, A. C., Liu, X., Bisaga, A., \& Nunes, E. V. (2006). Cognitive deficits predict low treatment retention in cocaine dependent patients. Drug and Alcohol Dependence, 81, 313-322.

Almeida, P. P., Novaes, M. A. F. P., Bressan R. A., \& Lacerda, A. L. T. (2008). Revisão: Funcionamento executivo e uso de maconha. Revista Brasileira de Psiquiatria, 30(1), 69-76.
American Psychiatric Association. (1994). Diagnostic and statistical manual of mental disorders ( $4^{\text {th }}$ ed.). Washington, DC: Author.

Amini, F., Alizadeh, H., \& Rezaee, O. (2012). Comparison of executive-neurological functions between addicted adults and normal adults. Annals of Biological Research, 3(1), 415-421.

Andrade, V. M., Santos, F. H., \& Bueno, O. F. A. (2004). Neuropsicologia hoje. São Paulo, SP: Artes Médicas.

Ballard, J. C. (2001). Assessing attention: Comparison of response inhibition and traditional continuous performance tests. Journal of Clinical and Experimental Neuropsychology, 23(3), 331-50.

Bechara, A., Dolan, S., Denburg, N., Hindes, A., Anderson, S. W., \& Nathan, P. E. (2001). Decision-making deficits, linked to a dysfunctional ventromedial prefrontal cortex, revealed in alcohol and stimulant abusers. Neuropsychology, 39, 376-389.

Berg, E. A. (1948). A simple objective test for measuring flexibility in thinking. Journal of General Psychology, 39, 15-22.

Bolla, K., Brown, K., Eldreth, D., Tate, K., \& Cadet, J. L. (2002). Dose-related neurocognitive effects of marijuana use. Neurology, 59, 1337-1343.

Campana, A. O. (1995). Introdução à investigação clínica. Botucatu, SP: Editora da Universidade Estadual Paulista Júlio de Mesquita Filho.

Carlini, E. A., Galduróz, J. C. F., Noto, A. R., \& Nappo, S. A. (2002). I Levantamento domiciliar sobre o uso de drogas psicotrópicas no Brasil. Brasília, DF: Secretaria Nacional Antidrogas.

Connolly, C. G., Foxe, J., Nierenberg, J., Shpaner, M., \& Garavan, H. (2012). The neurobiology of cognitive control in successful cocaine abstinence. Drug and Alcohol Dependence, $121,45-53$.

Cunha, J. A. (2000). Psicodiagnóstico-V (5. ed.). Porto Alegre, RS: Artes Médicas.

Cunha, P. J., \& Novaes, M. (2004). Avaliação neurocognitiva no abuso e dependência do álcool: Implicações para o tratamento. Revista Brasileira de Psiquiatria, 26(Supl. 1), 23-27.

Cunha, S. M., Peuker, A. C., \& Bizarro, L. (2012). Consumo de Álcool de Risco e Repertório de Habilidades Sociais entre Universitários. Psico, 43(3) 289-297.

Dougherty, D. M., Bjork, J. M., Harper, R. A., Marsh, D. M., Moeller, F. G., Mathias, C. W., \& Swann, A. C. (2003). Behavioral impulsivity paradigms: A comparison in hospitalized adolescents with disruptive behavior disorders. Journal of Child Psychology and Psychiatry, 44(8), 1145-1157.

Feil, J., Sheppard, D., Fitzgerald, P. B., Yücel, M., Lubman, D. I., \& Bradshaw, J. L. (2010). Addiction, compulsive drug seeking, and the role of frontostriatal mechanisms in regulating inhibitory control. Neuroscience and Biobehavioral Reviews, 35, 248-275.

Fillmore, M. T., \& Rush, C. R. (2002). Impaired inhibitory control of behavior in chronic cocaine users. Drug Alcohol Depend, 66(3), 265-273.

Finn, P. R., Mazas, C. A., Justus, A. N., \& Steinmetz, J. (2002). Early-onset alcoholism with conduct disorder: Go-no go learning deficits, working memory capacity, and personality. Alcoholism: Clinical and Experimental Research, 26(2), 186-206.

Forman, S. D., Dougherty, G. G., Casey, B. J., Siegle, G. J., Braver, T. S., Barch, D. M., ...Lorensen, E. (2004). Opiate addicts lack error-dependent activation of rostral anterior cingulate. Biological Psychiatry, 55(5), 531-537.

Goldstein, R. Z., \& Volkow, N. D. (2002). Drug addiction and its underlying neurobiological basis: Neuroimaging evidence for the involvement of the frontal cortex. The American Journal of Psychiatry, 159, 1642-1652. 
Goldstein, R. Z., Volkow, N. D., Wang, G. J., Fowler, J. S., \& Rajaram, S. (2001). Addiction changes orbitofrontal gyrus function: Involvement in response inhibition. Neuroreport, 12(11), 2595-2599.

Grafman, J. (1999). Experimental assessment of adult frontal lobe function. In B. L. Miller \& J. L. Cummings (Eds.), The human frontal lobes: Functions and disorders (pp. 321-344). New York: Guilford.

Heaton, K. R., Chelune, G. J., Talley, J. L., Kay, G. G., \& Curtiss, G. (1993). Wisconsin Card Sorting Test (WCST) Manual. Odessa, FL: Psychological Assessment Resources.

Heaton, K. R., Chelune, G. J., Talley, J. L., Kay, G. G., Curtiss, G., \& Cunha, J. A. (2005). Manual do teste Wisconsin de Classificação de Cartas. Revisado e Ampliado. Adaptação e Padronização Brasileira, Jurema Alcides Cunha e Cols. São Paulo: Casa do Psicólogo.

Hester, R., \& Garavan, H. (2004). Executive dysfunction in cocaine addiction: Evidence for discordant frontal, cingulate, and cerebellar activity. The Journal of Neuroscience, 24(49), 11017-11022.

Hutz, C. S., \& Nunes, C. H. (2001). Escala Fatorial de Ajustamento Emocional/ Neuroticismo - EFN. São Paulo, SP: Casa do Psicólogo.

Kamarajan, C., Porjesz, B., Jones, K. A., Choi, K., Chorlian, B. D., Padmanabhapillai, A., ...Begleiter, H. (2005). Alcoholism is a disinhibitory disorder: Neurophysiological evidence from a Go/No-Go task. Biological Psychology, 69, 353-373.

Kaufman, J. N., Ross, T. J., Stein, E. A., \& Garavan, H. (2003). Cingulate hypoactivity in cocaine users during a GO-NOGO task as revealed by event-related functional magnetic resonance imaging. The Journal of Neuroscience, 23(21), 7839-7843.

Kristensen, C. H. (2006). Funções executivas e envelhecimento. In M. A. M. P. Parente (Ed.), Cognição e envelhecimento (pp. 97-111). Porto Alegre, RS: Artmed.

Lezak, M. D. (1995). Neuropsychological assessment (3 ${ }^{\text {rd }}$ ed.). New York: Oxford University Press.

Li, C. S., Milivojevic, V., Kemp, K., Hong, K., \& Sinha, R. (2006). Performance monitoring and stop signal inhibition in abstinent patients with cocaine dependence. Drug Alcohol Depend, 85(3), 205-212.

Lundqvist, T. (2005). Cognitive consequences of cannabis use: Comparison with abuse of stimulants and heroin with regard to attention, memory and executive functions. Pharmacology Biochemistry and Behavior, 81(2), 319-330.

Moeller, F. G., Hasan, K. M., Steinberg, J. L., Kramer, L. A., Dougherty, D. M., Santos, R. M., ...Narayana, P. A. (2005). Reduced anterior corpus callosum white matter integrity is related to increased impulsivity and reduced discriminability in cocaine-dependent subjects: Diffusion tensor imaging. Neuropsychopharmacology, 30(3), 610-617.

Moss, M. B., \& Killiany, R. (1994). Neuroanatomical correlates of cognitive function. In J. M. Ellison, C. Weinstein, \& T. Hodel-Malinofsky (Eds.), The psychotherapist's guide to neuropsychiatry (pp. 23-72). Washington, DC: American Psychiatric Press.

Nations United Organization. (2012). Report of the International Narcotics Control Board for 2011. New York: Author.

Noel, X., Linden, M. V., Acremont, M., Colmant, M., Hanak, C., Peic, I., ...Bechara, A. (2005). Cognitive biases toward alcohol-related words and executive deficits in polysubstance abusers with alcoholism. Addiction, 100, 1302-1309.
Peuker, A. C., Lopes, F. M., Menezes, C. B., Cunha, S. M., \& Bizarro, L. (2013). Processamento Implícito e Dependência Química: Teoria, Avaliação e Perspectivas. Psicologia: Teoria e Pesquisa, 29(1), 07-14.

Riccio, C. A., Reynolds, C. R., Lowe, P., \& Moore, J. J. (2002). The continuous performance test: A window on the neural substrates for attention? Archives of Clinical Neuropsychology, 17(3), 235-272.

Rigoni, M. D. S., Susin, N., Trentini, C. M., \& Oliveira, M. D. S. (2012). Alcoolismo e Avaliação de Funções Executivas: Uma Revisão Sistemática. Psico, 44(1), 122-129.

Salamat, M. T., \& McPherson, D. L. (1999). Interactions among variables in the $\mathrm{P} 300$ response to a continuous performance task. Journal of American Academy of Audiology, 10(7), 379-87.

Schneider, E., \& Zuccoloto, A. (2007). E-prime 2.0 [Computer software]. Pittsburg, PA: Psychological Software Tools.

Strauss, E., Sherman, E. M. S., \& Spreen, O. (2006). A compendium of neuropsychological tests: Administration, norms, and commentary ( $3^{\text {rd }}$ ed.). New York: Oxford University Press.

Tinius, T. P. (2003). The intermediate visual and auditory continuous performance tests as a neuropsychological measure. Archives of Clinical Neuropsychology, 18(2), 199-214.

Turner, T. H., LaRowe, S., Horner, M. D., Herron, J., \& Malcolm, R. (2009). Measures of cognitive functioning as predictors of treatment outcome for cocaine dependence. Journal of Substance Abuse Treatment, 37, 328-334.

Verdejo-García, A., Bechara, A., Recknor, E. C., \& Pérez-García, M. (2006). Executive dysfunction in substance dependent individuals during drug use and abstinence: An examination of the behavioral, cognitive and emotional correlates of addiction. Journal of the International Neuropsychological Society, 12, 405-415.

Verdejo-García, A., Lopez-Torrecillas, F., Arcos, F. A., \& PerezGarcia, M. (2005). Differential effects of MDMA, cocaine, and cannabis use severity on distinctive components of the executive functions in polysubstance users: A multiple regression analysis. Addictive Behaviors, 30, 89-101.

Woicik, P. A., Moeller, S. J., Alia-Klein, N., Maloney, T., Lukasik, T. M., Yeliosof, O., ...Goldstein, R. Z. (2009). The neuropsychology of cocaine addiction: Recent cocaine use masks impairment. Neuropsychopharmacology, 34(5), 1112-1122.

Woicik, P. A., Urban, C., Alia-Klein, N., Henry, A., Maloney, T., Telang, F., ...Goldstein, R. Z. (2011). A pattern of perseveration in cocaine addiction may reveal neurocognitive processes implicit in the Wisconsin Card Sorting Test. Neuropsychologia, 49, 1660-1669.

World Health Organization. (1992). The ICD-10 Classification of Mental and Behavioral Disorders (CID10). Geneva, Switzerland: Author

Yucel, M., \& Lubman, D. I. (2007). Neurocognitive and neuroimaging evidence of behavioural dysregulation in human drug addiction: Implications for diagnosis, treatment and prevention. Drug and Alcohol Review, 26(1), 33-39. 